Clemons, T.D., Fitzgerald, M., Dunlop, S.A., Harvey, A.R., Iyer, K.S. \& Stubbs, K.A. (2013). An improved assay for the spectrophotometric determination of chondroitinase ABC activity. New Journal of Chemistry, 37(7), 1944-1949.

(C)The Royal Society of Chemistry and the Centre National de la Recherche Scientifique

This is pre-copy-editing, author-produced version of an article accepted for publication in New Journal of Chemistry following peer review. The definitive published version (see citation above) is located on the article abstract page of the publisher, Royal Society of Chemistry.

This version was made available in the UWA Research Repository on 8 April 2014 in compliance with the publisher's policies on archiving in institutional repositories.

Use of the article is subject to copyright law. 


\title{
An Improved Assay for the Spectrophotometric Determination of Chondroitinase ABC Activity
}

\author{
Tristan D. Clemons ${ }^{a}$, Melinda Fitzgerald ${ }^{b}$, Sarah A. Dunlop ${ }^{b}$, Alan R. Harvey ${ }^{c}$, K. Swaminathan Iyer*a, \\ and Keith A. Stubbs*a
}

\section{Received (in $X X X, X X X)$ Xth $X X X X X X X X X 20 X X$, Accepted $X$ th $X X X X X X X X X 20 X X$ DOI: $10.1039 / b 000000 x$}

Chondroitinase $\mathrm{ABC}$ (chABC) has evolved as a promising therapeutic for the degradation of inhibitory chondroitin sulfate proteoglycans (CSPGs) after injury to the central nervous system (CNS). The enzymatic breakdown of CSPGs by administered chABC reinstated plasticity as well as axon 10 regeneration; leading to improved CNS recovery. Thus in an effort to develop and evaluate treatments of CNS injury utilising chABC the accurate detection of levels of CSPGs and more importantly activity of chABC activity is of paramount importance. Here we present a spectrophotometric assay suitable for accurately and quantitatively measuring chABC activity, based on the use of 4-[3-(4-iodophenyl)-2-(4nitrophenyl)-2H-5-tetrazolio]-1,3-benzene disulfonate (WST-1). This novel WST-1 based assay was 15 compared to two other assays: measurement of alkene generation at $232 \mathrm{~nm}$ and a 1,9 dimethyl methylene blue (DMMB) assay which have been used previously for the detection of chABC activity and CSPGs respectively. Comparison of these assays demonstrated that the WST-1 assay was quantitative, efficient, cheap and easy to perform and overall was superior in the detection of chABC activity at all concentrations when compared to the other methods tested.

\section{${ }_{20}$ Introduction}

Chondroitin sulfate proteoglycans (CSPGs) are a diverse group of extracellular compounds consisting of a core protein and one or more covalently attached sulfated glycosaminoglycan (GAG) side chains. ${ }^{1}$ CSPGs play critical roles in a range of biological ${ }_{25}$ processes and diseases, providing a cellular framework to direct and at times inhibit cell growth and tissue formation within the extracellular matrix via activation of cell signalling pathways. ${ }^{2,3}$ Within the central nervous system (CNS) CSPGs play an important role in axon guidance during development as well as in 30 the formation and maturation of perineuronal nets (PNNs). ${ }^{3}$ Formation of PNNs has been well characterised to signal the maturation of synaptic connections and in turn the closing of the CNS's plastic phase. ${ }^{3-5}$ Following injury to the CNS a range of biochemical changes occur, including a sharp rise in the levels of
${ }_{35}$ CSPGs associated with the formation of the glial scar at the primary injury. ${ }^{4}$ It has been shown that the presence of CSPGs at the site of injury results in an environment that is inhibitory to axon regeneration and axonal plasticity, two important factors required for restoration of function post-CNS injury. ${ }^{1,4}$ 40 Chondroitinase $\mathrm{ABC}$ (chABC), has been used to successfully degrade CSPGs and PNN structures at the site of a CNS injury both in vitro and in vivo, which in turn resulted in axon regeneration and more importantly the reintroduction of plasticity at the injury site. ${ }^{1,4,6}$ Despite this proof-of-concept demonstration 45 of the use of chABC in treating CNS injury, several limitations have been reported.

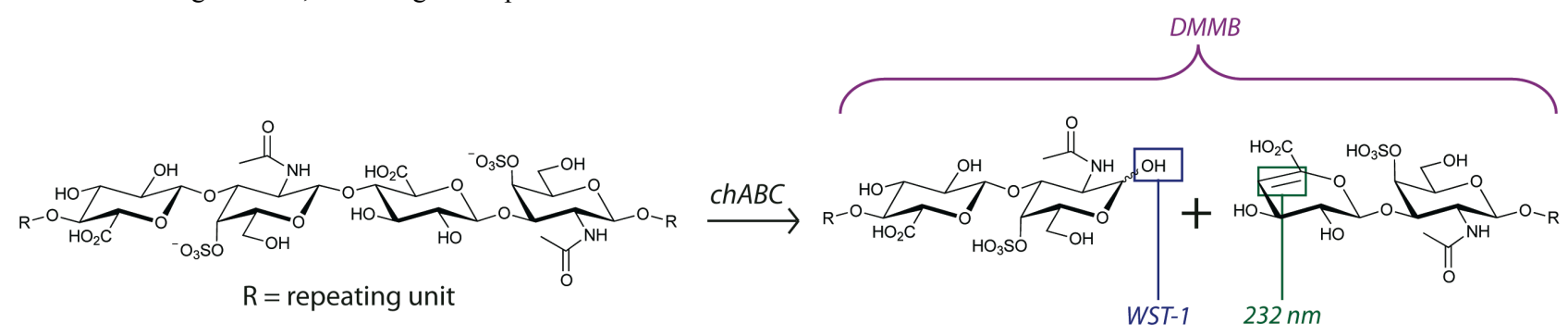

Figure 1. chABC cleavage of chondroitin sulfate A to produce the reducing disaccharide (detected by WST-1) and the 4,5-unsaturated disaccharide 50 product (detected by measuring the absorbance at $232 \mathrm{~nm}$ ). DMMB will bind to large intact proteoglycans. 
The most notable of these is that the enzyme is thermally sensitive and almost all of its activity is lost within 3-5 days at 37 ${ }^{\circ} \mathrm{C}$. ${ }^{7}$ In order to develop better methods of delivery of chABC, given the potential of chABC as a therapeutic intervention, the 5 ability to quantify and accurately assay its activity in model systems is of paramount importance. This is especially important in comparing efficacy of treatments given the broad range in the persistence of enzyme activity seen with commercially purchased chABC batches.?

10 The enzyme commonly used in these studies is chABC from Proteus vulgaris. It has broad substrate specificity degrading chondroitin, chondroitin-4-sulfate (also known as chondroitin sulfate A, CS-A), chondroitin-6-sulfate, dermatan sulfate, and hyaluronan by $\beta$-elimination of 1,4-hexosaminidic bonds to 15 produce $\Delta 4,5$-unsaturated disaccharides and a reducing end polysaccharide $^{8,9}$ (Figure 1), overall acting as a GAG lyase. Methods previously used for the measurement of chABC activity have been largely based around the use of radioactive substrates, ${ }^{9}$ FACE,${ }^{10,11}$ HPLC $^{7,12}$ or through indirect detection methods using 20 metachromatic dyes such as Alcian Blue or 1,9dimethylmethylene blue (DMMB). ${ }^{13-16}$ These techniques have been used for the analysis of CSPGs in urine samples, cartilage culture media, mast cell heparin and chromatographic column sections containing GAGs and proteoglycans. ${ }^{14}$ Methodologies 25 based around the use of dyes such as Alcian blue or more recently DMMB have been the most popular assays used for chABC detection, due to their relative speed, ease of use and safety. However despite these advantages, these methods suffer from limitations. The measurements obtained give amounts of total 30 sulfated GAGs present, and therefore are an indirect measurement of chABC activity. These assays also are affected by polyanions, including other sulfated GAGs, DNA and RNA, resulting in positive interference. Furthermore, there are limitations of these assays surrounding their quantitative 35 detection limits and stability of the formed complexes. ${ }^{14,15} \mathrm{~A}$ further method that has been used for detection of chABC activity involves measuring the formation of the C4-C5 double bond in the product material at $232 \mathrm{~nm}$ (Figure 1). Unfortunately, this method also suffers from problems associated with potential

40 interferences at this wavelength and these problems are compounded by the fact that alkenes are relatively weak chromophores. ${ }^{9}$

One method that has received some attention for the detection of biological products ${ }^{17}$ is the use of tetrazolium salts that 45 produce highly coloured formazan dyes under reducing conditions. The compound of choice, 4-[3-(4-iodophenyl)-2-(4nitrophenyl)-2H-5-tetrazolio]-1,3-benzene disulfonate (WST-1), is used in cell metabolism kits (Roche), cell viability assays ${ }^{18}$ and for measuring anti-inflammatory responses. ${ }^{19,20}$ To date though, ${ }_{50}$ the use of this system in assaying for carbohydrate-processing enzyme activity has only received limited attention. ${ }^{21}$ The use of WST-1 has advantages in that it is a direct measure of enzyme activity due to its ability to form water-soluble complexes that result in a stable complex suitable for accurate 55 spectrophotometric measurements. Spectrophotometric detection is achieved through direct incubation of the WST-1, which in turn reacts with the newly formed reducing disaccharide found only in the product (Figure 1), reducing the WST-1 to WST-1 formazan. ${ }^{21}$ To date however a rigorous investigation into the use ${ }_{60}$ of WST-1 for measuring chABC activity has not been conducted. If this method was amenable to measuring chABC activity then a potentially more sensitive and accurate measurement of chABC would be available that could be used to give valuable insight into developing methods for delivery of chABC for treating CNS ${ }_{65}$ injury. Here we compare three assays used for chABC activity measurements, demonstrating that WST-1 is a viable, sensitive and reliable alternative to the more classical methods for the measurement of chABC activity in model systems.

\section{Results and Discussion}

70 The three spectrophotometric assays tested included the use of DMMB for the measurement of intact CSPGs present, the evolution of alkenes and in turn the absorbance signal at $232 \mathrm{~nm}$ produced from enzymatic activity and finally the novel WST-1 based assay. Initially the assays were compared for their 75 sensitivity to changes in the concentration of chABC against a fixed substrate concentration (chondroitin sulfate A, CS-A, 10 $\mu \mathrm{M})$ and time of incubation (2 hours). Treatment of CS-A with a range of chABC concentrations resulted in contrasting results. It was clearly evident that the response for the WST-1 assay was of 80 higher order with regards to linearity and response sensitivity in comparison to both the $232 \mathrm{~nm}$ assay and the DMMB assay. This was indicated by the strongest linear correlation coefficient as well as the largest response to increases in enzyme concentration (Figure 2). This linearity is presumably a result of the newly ${ }_{85}$ formed hemiacetal from the chABC reaction directly reducing the WST-1 to its coloured formazan (Figure 1). The steeper gradient suggests the range and sensitivity of the WST-1 assay was superior to the other two assays compared in this study.

A.

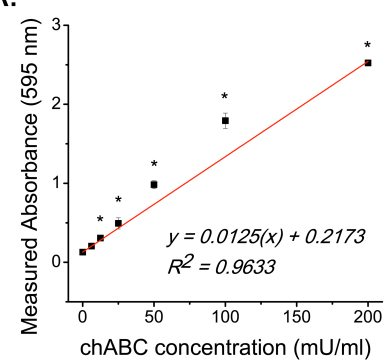

B.

C.
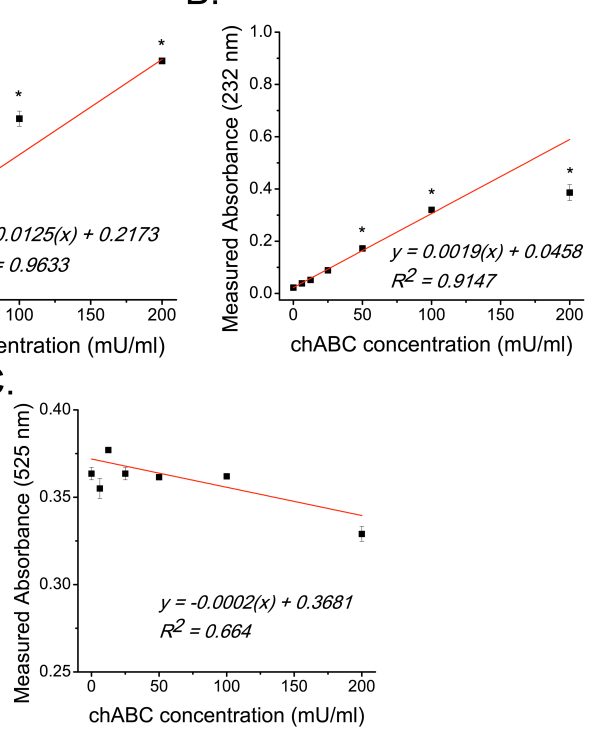

Figure 2. ChABC standard curves measured with variable enzyme 90 concentration and a constant CS-A concentration of $10 \mu \mathrm{M}$. A) WST-1 assay, B) Absorbance at $232 \mathrm{~nm}$, C) DMMB assay. Results are shown as mean \pm standard deviation of three replicates, significance from background levels determined by ANOVA and Bonferroni-Dunn post-hoc tests (Significance of $\mathrm{P} \leq 0.05$.).

95 A more rigorous statistical analysis (Bonferroni-Dunn post-hoc 
test $(\mathrm{P} \leq 0.05))$ was performed to determine at which concentration each assay could detect a significant difference from the background i.e. no enzyme present in the system. Using the WST-1 assay a significant difference could be detected at an 5 enzyme concentration of $12.5 \mathrm{mU} / \mathrm{ml}$, whilst detection using the absorbance at $232 \mathrm{~nm}$ assay only was significantly different from background at $50 \mathrm{mU} / \mathrm{ml}$. Surprisingly the DMMB assay showed no significant difference from background levels even at 200 $\mathrm{mU} / \mathrm{ml}$, despite an apparent trend in the data (Figure 2).

10 In an effort to confirm the reliability of the WST-1 assay against a variety of enzyme concentrations, similar experiments were conducted at a range of chABC concentrations over increasing incubation times. The rate of product formation and in turn chABC catalysed CS-A cleavage was linear for 15 concentration ranges between $5-50 \mathrm{mU} / \mathrm{ml} \mathrm{chABC}$ for up to 6 hours of digestion (Figure 3). These results indicate that the assay is not substrate limiting or experiencing any feedback inhibition as a result of product accumulation within the system. Furthermore the direct relationship evident for all concentrations 20 tested indicates, as expected, that the cleavage of CS-A is proportional to the amount of chABC present in the system.

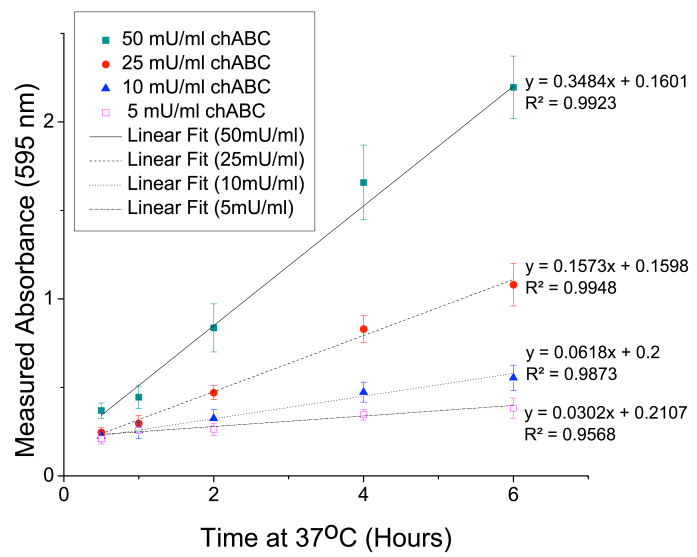

Figure 3. ChABC does not experience substrate inhibition as measured using the WST-1 assay. Varying chABC concentrations against a constant 25 substrate concentration $(\mathrm{CS}-\mathrm{A}, 20 \mu \mathrm{M})$. Results are shown as mean \pm standard deviation of three replicates.

With the lower limits of detection of enzyme activity against a fixed substrate concentration determined, we felt it was important to measure assay sensitivity with samples containing small 30 amounts of substrate (CS-A). We incubated a range of low CS-A concentrations $(0.01-0.5 \mu \mathrm{M})$ with a fixed concentration of chABC $(50 \mathrm{mU} / \mathrm{ml})$. The concentration of enzyme chosen was based on its ability to produce significant digestion after 2 hours of incubation when tested with the WST-1 assay, the absorbance 35 at $232 \mathrm{~nm}$ assay and the DMMB assay. Once again the WST-1 based assay provided the best sensitivity (Figure 4A). Using the same statistical analysis as above we found that no values tested in either the DMMB assay or by measuring the absorbance of samples at $232 \mathrm{~nm}$ were significantly above baseline levels

40 (Figure 4B,C). The WST-1 assay however showed significance at all concentrations of substrate greater then $0.05 \mu \mathrm{M}$ CS-A.
45 Table 1. Comparison of chABC kinetic parameters. Values are calculated as mean \pm standard deviation from triplicate measurements. Prabhakar et al. data is given in Reference 22 .

\begin{tabular}{ccc}
\hline Assay method & $\boldsymbol{K}_{\mathbf{m}}(\boldsymbol{\mu M})$ & $\boldsymbol{k}_{\text {cat }}\left(\mathbf{m i n}^{-1}\right)$ \\
\hline WST-1 Assay & $8.01 \pm 0.99$ & $3123 \pm 456$ \\
\hline 232 nm assay & $14.85 \pm 7.26$ & $3848 \pm 1339$ \\
& $(1.5 \pm 0.1)^{22}$ & $(52000 \pm 1300)^{22}$ \\
\hline
\end{tabular}

Now that we had demonstrated the lower detection limits of so the enzyme and substrate at fixed substrate and enzyme concentrations respectively, we felt it was important to determine the usefulness of the WST-1 assay for assessing kinetic parameters of the enzyme. This is important from the perspective of using chABC as a therapeutic intervention against CNS injury 55 as differences between enzyme batches and substrate preference can be determined prior to use, as well as enzyme activity can be monitored in time course experiments. Due to its mode of action the WST-1 assay has the advantage that it can be used for the measurement of chABC kinetic parameters. Using this assay the 60 substrate dependence of chABC activity was investigated and found to conform to Michaelis-Menten kinetics (Figure 5). The $K_{\mathrm{m}}(8.01 \pm 0.99 \mu \mathrm{M})$ determined using the WST-1 assay was within reasonable comparison to values determined by the absorbance at $232 \mathrm{~nm}$ method and those published within the ${ }_{65}$ literature (Table 1).
A.

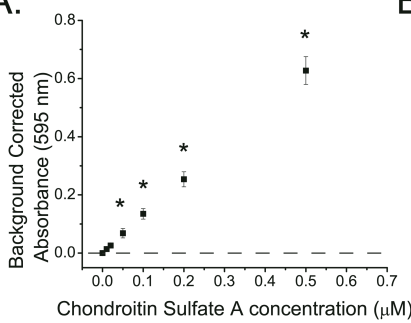

C.

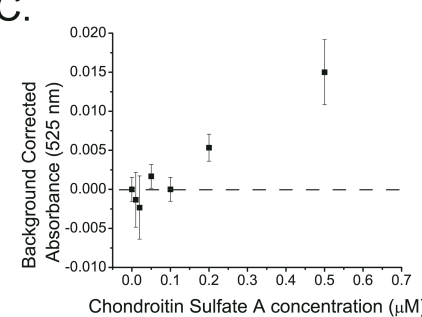

Figure 4. Comparison of assay substrate detection limits with CS-A. A range of low substrate concentrations (CS-A, $0.01-0.5 \mu \mathrm{M})$ were incubated with a fixed chABC concentration $(50 \mathrm{mU} / \mathrm{ml})$, with substrate digestion measured by A) WST-1 assay, B) Absorbance at $232 \mathrm{~nm}$ assay 70 and C) DMMB assay. Results are shown as mean \pm standard deviation of three replicates. Significance of the results against background levels was determined with ANOVA and Bonferroni-Dunn post-hoc tests (Significance of $\mathrm{P} \leq 0.05$.)

However the values for the $k_{\text {cat }}$ determined by the WST-1 75 assay $\left(3123 \pm 456 \mathrm{~min}^{-1}\right)$ and by the absorbance at $232 \mathrm{~nm}(3848$ $\pm 1339 \mathrm{~min}^{-1}$ ) method for the chABC sample used in this study was much lower then those published previously using the absorbance signal at $232 \mathrm{~nm}$ method (Table 1). ${ }^{22}$ The differences in $k_{\text {cat }}$ could be attributed to the fact that the previous study 
assessed a highly purified and freshly prepared chABC sample using the absorbance at $232 \mathrm{~nm}$ assay in comparison to the chABC used in this study. A limitation in the DMMB assay is that due to its method for determining activity of chABC it is 5 unsuitable for the determination of kinetic parameters for the enzyme and hence was not compared in these experiments.

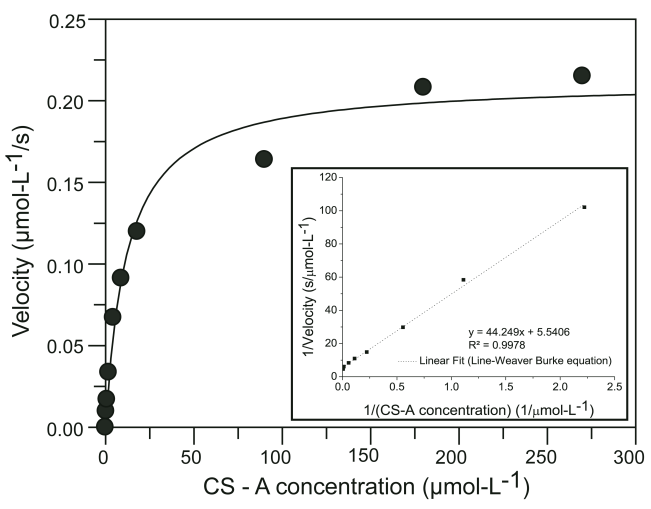

Figure 5. Michaelis-Menten kinetic analysis (inset Lineweaver-Burk plot) of chABC with CS-A as substrate using the WST-1 based assay.

Although strong evidence of therapeutic benefit has been 10 reported using chABC for treatment of CNS injury, questions remain with regards to the persistence of enzyme activity at 37 ${ }^{\circ} \mathrm{C} .{ }^{7,23}$ The disaccharide trehalose has long been shown to have a positive effect on enzyme stability in both resisting denaturation due to heat treatment as well as during the process of 15 lyophilisation. ${ }^{24,25}$ Despite considerable debate with regards to the mechanism by which trehalose is able to stabilise enzymes, ${ }^{25-27}$ positive effects have been seen with this compound and chABC. ${ }^{23}$

A.

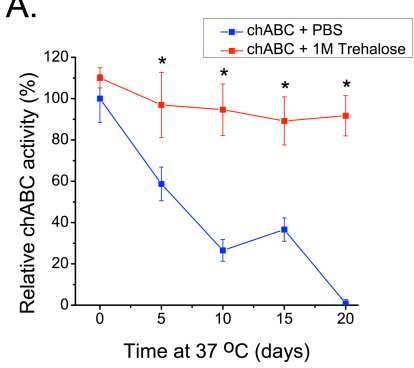

B.

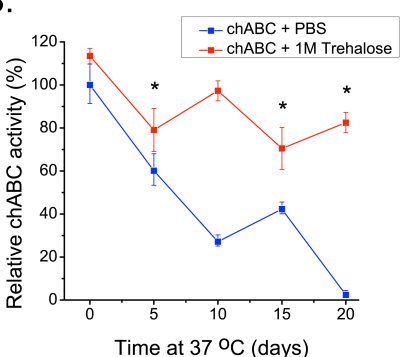

C.

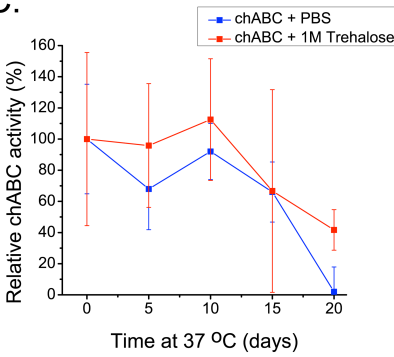

Figure 6. Stabilising effects on chABC due to $1 \mathrm{M}$ trehalose. Activity of samples measured by A) WST-1 assay, B) Absorbance measured at 232 $20 \mathrm{~nm}, \mathrm{C})$ DMMB assay. Results are shown as mean \pm standard deviation of three replicates. Statistical analysis was performed to test significance of means differing at each time point tested with an ANOVA and Bonferroni-Dunn post-hoc test $(\mathrm{P} \leq 0.05)$.

Here we tested the ability of $1 \mathrm{M}$ trehalose to stabilise chABC ${ }_{25}$ at $37{ }^{\circ} \mathrm{C}$ and improve persistence of activity, comparing each of the assays for the ability to detect any loss in activity over time (Figure 6). It was evident that $1 \mathrm{M}$ trehalose significantly preserved chABC activity at $37{ }^{\circ} \mathrm{C}$, consistent with findings reported in the literature ${ }^{23}$ and evident to varying degrees in each 30 of the assays compared (Figure 6). This was also confirmed by SDS-PAGE using decorin as a substrate to directly show the degradation of sulfated GAG side chains from a core protein (Supporting Figures S1 and S2). Once again the best sensitivity was seen with the WST-1 assay which showed significant 35 stabilisation from day 5 onwards when comparing the trehalose stabilised samples to the control chABC at the same time points. The measurement of alkene formation at $232 \mathrm{~nm}$ showed a similar result but with significant stabilisation only detected from day 10 onwards. Interestingly, using the DMMB assay resulted in

40 similar trends but no conclusive significant differences were observed between the trehalose stabilised samples and that of the control chABC samples, despite clear evidence from the SDSPAGE experiments that demonstrate loss of chABC activity in the absence of trehalose (Supporting information S1, S2).

\section{${ }_{45}$ Conclusions}

The use of chABC has been demonstrated to be a potentially viable therapeutic option for degrading CSPGs at the site of a CNS injury. ${ }^{6,23,28}$ As a result, useful biochemical assays need to be developed to measure the effectiveness of the enzyme in a 50 variety of conditions. We have demonstrated that the water soluble tetrazolium dye WST-1 is an effective reagent for the detection of reducing end sugars produced by chABC induced enzymatic cleavage of suitable biological substrates. In comparison to other spectrophotometric assays used for the 55 measurement of chABC activity, the WST-1 based assay provides superior results with regards to response and sensitivity at low levels of enzyme and substrate. This assay can also be used to measure the kinetic parameters of chABC as well as the direct thermal stabilisation of chABC in the presence of trehalose.

${ }_{60}$ The WST-1 assay may be overall a more general strategy and will find utility in the analyses of chABC activity for industrial and biotechnological purposes.

\section{Experimental}

\section{Materials}

${ }_{65}$ Chondroitinase ABC (chABC) from Proteus vulgaris, chondroitin sulfate A from bovine trachea, trehalose, 1,9 dimethyl methylene blue (DMMB) were used as received from Sigma Aldrich. The tetrazolium salt WST-1 [2-(4-indophenyl)-3(4-nitrophenyl)-5-(2,4-disulfophenyl)-2H tetrazolium, 70 monosodium salt] was used as received from Santa Cruz Biotechnology, inc.

\section{WST-1 chABC assay}

Assay conditions consisted of $90 \mu \mathrm{l}$ substrate at the desired concentration (CS-A prepared in PBS $\mathrm{pH} 7.4,0.01 \mu \mathrm{M}-270$ $75 \mu \mathrm{M})$ with $10 \mu \mathrm{chABC}(5 \mathrm{mU} / \mathrm{ml}-200 \mathrm{mU} / \mathrm{ml})$ added at $\mathrm{t}=0$. Samples were incubated for 2 hours at $37{ }^{\circ} \mathrm{C}$ before being quenched with $100 \mu \mathrm{l}$ WST-1 working reagent $(1.1 \mathrm{mg} / \mathrm{ml}$ WST1 prepared in $0.1 \mathrm{M} \mathrm{NaOH})$. Samples were then transferred to an incubator at $60{ }^{\circ} \mathrm{C}$ for 1 hour to allow for colour development 
before measuring the absorbance of the samples in a 96-well plate at $595 \mathrm{~nm}$ on an Enspire 2300 multimodal plate reader.

\section{DMMB chABC assay}

Assay conditions consisted of $90 \mu \mathrm{l}$ substrate at desired 5 concentration (CS-A prepared in PBS pH 7.4, $0.01 \mu \mathrm{M}-270$ $\mu \mathrm{M})$ with $10 \mu \mathrm{l} \operatorname{chABC}(5 \mathrm{mU} / \mathrm{ml}-200 \mathrm{mU} / \mathrm{ml})$ added at $\mathrm{t}=0$. Samples were incubated for 2 hours at $37{ }^{\circ} \mathrm{C}$ before $50 \mu \mathrm{l}$ of reaction mixture was transferred to wells of a $96-$ well plate. To each well, $150 \mu \mathrm{l}$ of DMMB working reagent was added 10 (prepared according to previously described methods ${ }^{14}$ ) and the absorbance at $525 \mathrm{~nm}$ was immediately measured within 15 seconds of the addition of working reagent.

\section{Absorbance at $232 \mathrm{~nm}$ chABC assay}

Assay conditions consisted of $90 \mu \mathrm{l}$ substrate at desired 15 concentration (CS-A prepared in PBS pH 7.4, $0.01 \mu \mathrm{M}-270$ $\mu \mathrm{M})$ with $10 \mu \mathrm{chABC}(5 \mathrm{mU} / \mathrm{ml}-200 \mathrm{mU} / \mathrm{ml})$ added at $\mathrm{t}=0$. Samples were incubated for 2 hours at $37{ }^{\circ} \mathrm{C}$ before being heat treated at $90{ }^{\circ} \mathrm{C}$ for 10 minutes to halt the reaction. Sample absorbance was then measured using a NanoDrop UV-Vis 20 instrument set at $232 \mathrm{~nm}$.

\section{Substrate inhibition experiment}

chABC at varying concentrations $(10 \mu 1$ at $5,10,25,50 \mathrm{mU} / \mathrm{ml})$, was combined with $90 \mu 1$ of $20 \mu \mathrm{M}$ CS-A at $\mathrm{t}=0$ hours. Samples ${ }_{25}$ were incubated at $37^{\circ} \mathrm{C}$ for $0.5,1,2,4$ or 6 hours before being quenched with WST-1 working reagent and assayed for activity as described above. All assays were performed in triplicate.

\section{Kinetic analysis WST-1}

${ }_{30}$ Concentrations of CS-A were prepared in PBS pH 7.4 and varied from $0-270 \mu \mathrm{M}$ with $90 \mu \mathrm{l}$ of solution per tube. chABC $(10 \mu \mathrm{l}$, $50 \mathrm{mU} / \mathrm{ml}$ ) prepared in PBS $\mathrm{pH} 7.4$ was added to each tube in a staggered arrangement to ensure a consistent time of incubation for each sample. Samples were incubated together for 2 hours at $3537{ }^{\circ} \mathrm{C}$ before being quenched with WST-1 and assayed for activity according to the procedure outlined above. Path length was determined based on manufacturers dimensions of the 96 well-plate (Greiner Bio-One Cell Star), with the WST-1 extinction coefficient calculated from a standard curve 40 (Supporting information S3.)

\section{ChABC versus trehalose treated chABC}

ChABC samples of final concentration $200 \mathrm{mU} / \mathrm{ml}$ were either prepared in PBS pH 7.4 (chABC control) or in $1 \mathrm{M}$ trehalose ${ }_{45}$ solution in PBS $\mathrm{pH} 7.4$, from a chABC stock of $10 \mathrm{U} / \mathrm{ml}$. Samples were heat treated at $37^{\circ} \mathrm{C}$ for $0.5,10,15$ or 20 days and then were immediately frozen $\left(-40{ }^{\circ} \mathrm{C}\right)$. The samples were then thawed together on ice and assayed for activity following the three protocols described above. For analysis of activity against ${ }_{50}$ decorin, $10 \mu \mathrm{l}$ aliquots of the enzyme samples were combined with decorin $(10 \mu \mathrm{l}, 800 \mu \mathrm{g} / \mathrm{ml}, 8 \mu \mathrm{g})$ and incubated together at $37^{\circ} \mathrm{C}$, for 4 hours. After reaction the samples where prepared and separated on a $10 \%$ SDS-PAGE gel following standard protocols.

\section{Acknowledgements}

55 This work was funded by the Australian Research Council (ARC), and the National Health \& Medical Research Council (NHMRC).

\section{Notes and references}

${ }^{a}$ School of Chemistry and Biochemistry, The University of Western 60 Australia, Crawley, WA, 6009, Australia. E-mail: swaminatha.iyer@uwa.edu.au; $\quad+61 \quad$ 6488-4470; keith.stubbs@uwa.edu.au; Tel: +616488-2725

${ }^{b}$ Experimental and Regenerative Neurosciences, School of Animal Biology, The University of Western Australia, Crawley, WA, 6009, 65 Australia.

${ }^{c}$ School of Anatomy, Physiology and Human Biology, The University of Western Australia, Crawley, WA, 6009, Australia.

$\dagger$ Electronic Supplementary Information (ESI) available: Activity loss of chABC samples against decorin either with or without trehalose stabilised 70 enzyme assessed by SDS-PAGE and WST-1 standard curve used for the determination of the molar absorptivity of WST-1 at $595 \mathrm{~nm}$. See DOI: $10.1039 / \mathrm{b} 000000 \mathrm{x} /$

1. G. Garcia-Alias and J. W. Fawcett, Exp. Neurol., 2012, 235, 26.

752 . T. Laabs, D. Carulli, H. Geller and J. Fawcett, Curr. Opin. Neurobiol., 2006, 15, 116.

3. C. Galtrey and J. Fawcett, Brain Res. Rev., 2007, 54, 1.

4. J. Kwok, F. Afshari, G. Garcia-Alias and J. Fawcett, Restor. Neurol. Neurosci., 2008, 26, 131.

80 5. T. Pizzorusso, P. Medini, N. Berardi, S. Chierzi, J. Fawcett and L. Maffei, Science, 2002, 298, 1248.

6. E. Bradbury, L. Moon, R. Popat, V. King, G. Bennett, P. Patel, J. Fawcett and S. McMahon, Nature, 2002, 416, 636.

7. N. J. Tester, A. H. Plaas and D. R. Howland, J. Neurosci. Res., 2007, $85 \mathbf{8 5}, 1110$

8. W. Huang, V. Lunin, Y. Li, S. Suzuki, N. Sugiura, H. Miyazono and M. Cygler, J. Mol. Biol., 2003, 328, 623.

9. T. Yamagata, H. Saito, O. Habuchi and S. Suzuki, J. Biol. Chem., 1968, 243, 1523.

90 10. A. Calabro, V. C. Hascall and R. J. Midura, Glycobiology, 2000, 10, 283.

11. Y. Oonuki, Y. Yoshida, Y. Uchiyama and A. Asari, Anal. Biochem., 2005, 343, 212.

12. N. Volpi, Anal. Biochem., 2000, 277, 19.

95 13. E. Gold, Anal. Biochem., 1979, 99, 183.

14. R. W. Farndale, D. J. Buttle and A. J. Barrett, Biochim. Biophys. Acta., 1986, 883, 173.

15. I. Barbosa, S. Garcia, V. Barbier-Chassefiere, J. P. Caruelle, I. Martelly and D. Papy-Garcia, Glycobiology, 2003, 13, 647.

100 16. C. B. Whitley, M. D. Ridnour, K. A. Draper, C. M. Dutton and J. P. Neglia, Clin. Chem., 1989, 35, 374.

17. M. Ishiyama, M. Shiga, K. Sasamoto, M. Mizoguchi and P. G. He, Chem. Pharm. Bull., 1993, 41, 1118.

18. J. Ranke, K. Molter, F. Stock, U. Bottin-Weber, J. Poczobutt, J. Hoffmann, B. Ondruschka, J. Filser and B. Jastorff, Ecotoxicol. Environ. Saf., 2004, 58, 396.

19. A. S. Tan and M. V. Berridge, J. Immunol. Methods, 2000, 238, 5968.

20. R. Mullings and J. H. Parish, Enzyme Microb. Technol., 1984, 6, 491. 110 21. E. Hammond, C. P. Li and V. Ferro, Anal. Biochem., 2010, 396, 112.

22. V. Prabhakar, I. Capila, C. J. Bosques, K. Pojasek and R. Sasisekharan, Biochem. J., 2005, 386, 103.

23. H. Lee, R. McKeon and R. Bellamkonda, Proc. Nat. Acad. Sci. USA, 2010, 107, 3340.

115 24. K. Lippert and E. Galinski, Appl. Microbiol. Biot., 1992, 37, 61.

25. M. Sola-Penna, J. Roberto and J. Meyer-Fernandes, Arch. Biochem. Biophys., 1998, 360, 10.

26. M. Mazzobre, M. Pilar Buera and J. Chirife, Lebensm. Wiss. Technol., 1997, 30, 324.

120 27. J. Kaushik and R. Bhat, J. Biol. Chem., 2005, 278, 26458.

28. R. Lin, J. Kwok, D. Crespo and J. Fawcett, J. Neurochem., 2008, 104, 400. 


\section{An Improved Assay for the Spectrophotometric Determination of Chondroitinase ABC Activity}

Tristan D. Clemons ${ }^{a}$, Melinda Fitzgerald ${ }^{b}$, Sarah A. Dunlop ${ }^{b}$, Alan R. Harvey ${ }^{c}$, K. Swaminathan Iyer*a, and Keith A. Stubbs* ${ }^{* a}$

Supporting Information

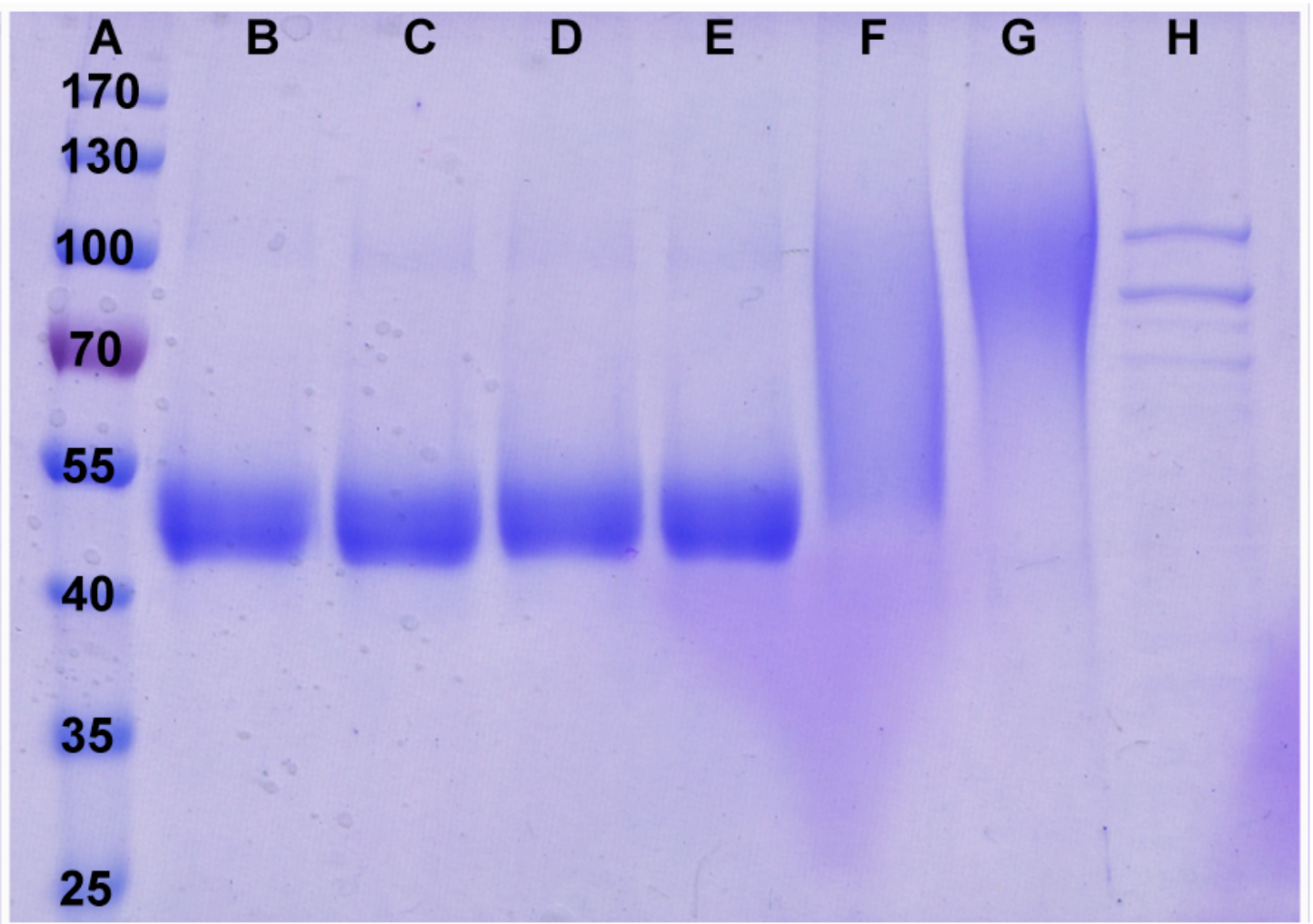

Figure S1 - SDS-PAGE analysis of degradation products from heat treated chABC alone, with decorin as substrate (i.e. no trehalose added). A) Ladder, B) 0 days, C) 5 days, D) 10 days, E) 15 days, F) 20 days chABC heat treatment at $37^{\circ} \mathrm{C}$, G) decorin with PBS substrate control and $\mathrm{H}$ ) chABC with PBS control. Dense bands at approximately $55 \mathrm{kDa}$ correlate to the decorin core protein. A diffuse band correlates to the native sulphated glycosaminoglycan side chain decorated core protein illustrated by lane $\mathrm{F}$ and $\mathrm{G}, 20$ days of chABC heat treatment and control decorin respectively. 


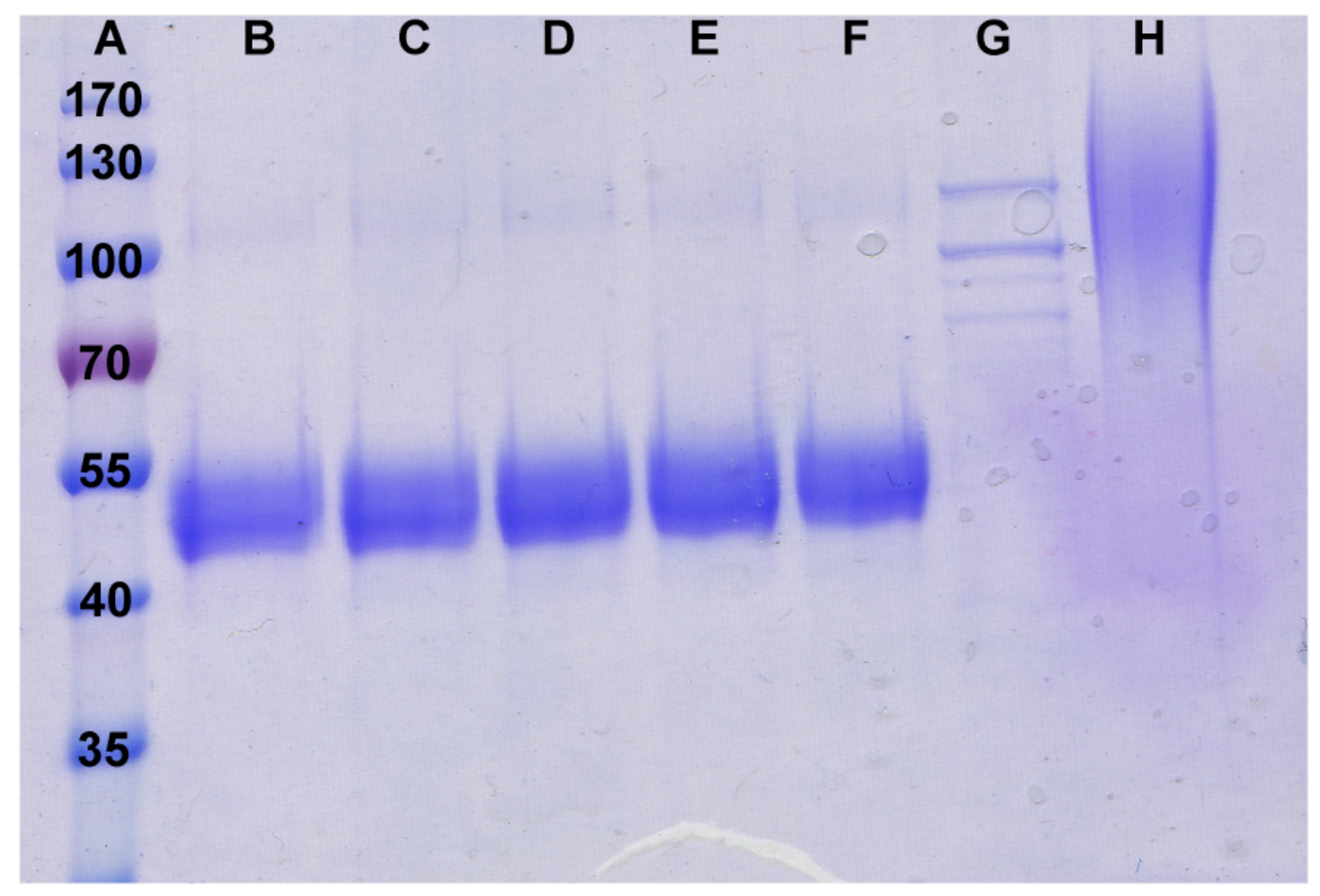

Figure S2 - SDS-PAGE analysis of degradation products from heat treated chABC in the presence of trehalose, with decorin as substrate. A) Ladder, B) 0 days, C) 5 days, D) 10 days, E) 15 days, F) 20 days chABC + trehalose heat treatment at $37{ }^{\circ} \mathrm{C}, \mathrm{G}$ ) chABC with trehalose control and $\mathrm{H}$ ) decorin with trehalose substrate control. Dense bands at approximately $55 \mathrm{kDa}$ correlate to the decorin core protein. A diffuse band correlates to the native sulphated glycosaminoglycan side chain decorated core protein illustrated by lane $\mathrm{H}$ decorin + trehalose control. 


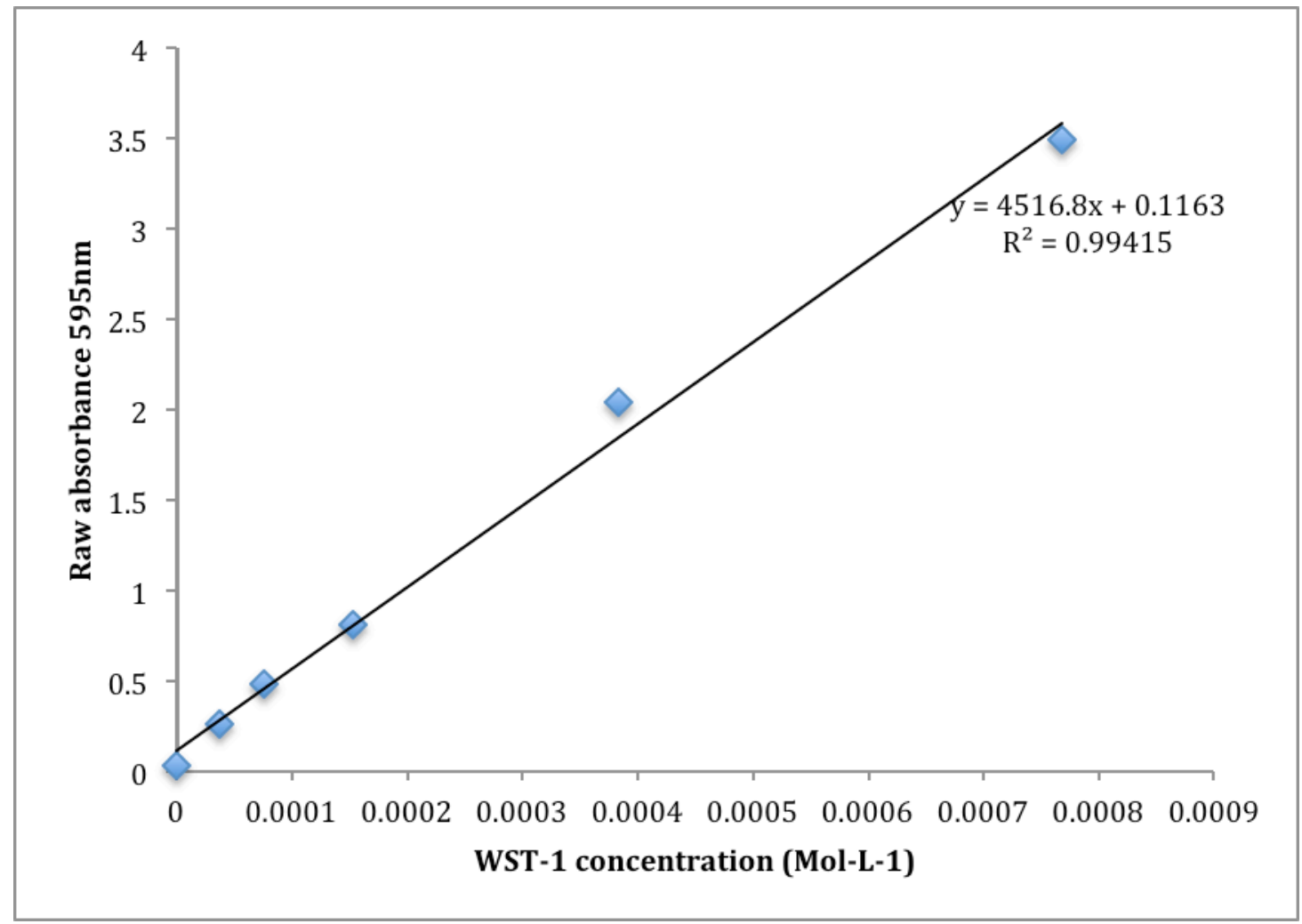

Figure S3 - Standard curve used for the calculation of WST-1 molar absorption coefficient. 